\title{
The Molecular Tweezer CLR01 Stabilizes a Disordered Protein- Protein Interface
}

\author{
David Bier, ${ }^{\dagger,}$ Sumit Mittal, ${ }^{\S, \#}$ Kenny Bravo-Rodriguez, ${ }^{\S, \#}$ Andrea Sowislok, ${ }^{\ddagger, \#}$ Xavier Guillory, ${ }^{\dagger, \ddagger}$ \\ Jeroen Briels, ${ }^{\dagger,}$ Christian Heid, ${ }^{\ddagger}$ Maria Bartel, ${ }^{\dagger}$ Burkhard Wettig, ${ }^{\ddagger}$ Luc Brunsveld, ${ }^{\dagger}$

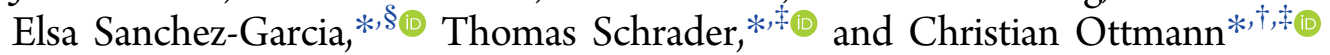

${ }^{\dagger}$ Laboratory of Chemical Biology, Department of Biomedical Engineering and Institute for Complex Molecular Systems, Eindhoven University of Technology, Den Dolech 2, 5612 AZ Eindhoven, The Netherlands

${ }^{\ddagger}$ Department of Chemistry, University of Duisburg-Essen, Universitätsstrasse 7, 45117 Essen, Germany

${ }^{\S}$ Max-Planck-Institut für Kohlenforschung, Kaiser-Wilhelm-Platz 1, 45470 Mülheim an der Ruhr, Germany

Supporting Information

ABSTRACT: Protein regions that are involved in proteinprotein interactions (PPIs) very often display a high degree of intrinsic disorder, which is reduced during the recognition process. A prime example is binding of the rigid 14-3-3 adapter proteins to their numerous partner proteins, whose recognition motifs undergo an extensive disorder-to-order transition. In this context, it is highly desirable to control this entropy-costly process using tailored stabilizing agents. This study reveals how the molecular tweezer CLR01 tunes the 14$3-3 / \mathrm{Cdc} 25 \mathrm{CpS} 216$ protein-protein interaction. Protein crystallography, biophysical affinity determination and biomolecular simulations unanimously deliver a remarkable finding: a supramolecular "Janus" ligand can bind simultaneously to a flexible peptidic PPI recognition motif and to a well-structured adapter protein. This binding fills a gap in the protein-protein interface, "freezes" one of the conformational states of the intrinsically disordered $\mathrm{Cdc} 25 \mathrm{C}$ protein partner and enhances the apparent affinity of the interaction. This is the first structural and functional proof of a supramolecular ligand targeting a PPI interface and stabilizing the binding of an intrinsically disordered recognition motif to a rigid partner protein.

\section{INTRODUCTION}

Intrinsically disordered proteins (IDPs) play a major role in almost every aspect of biology, ranging from signal transduction over cell cycle control, translation and transcription to a variety of different diseases. ${ }^{1,2}$ About $30 \%$ of eukaryotic proteomes are proposed to be disordered. ${ }^{3}$ In principle, IDPs can be described as complex molecules that populate ensembles of diverse, this behavior for protein-protein interactions (PPIs) is the ability of a specific motif to bind multiple partner proteins, each employing a different conformation of the recognition motif. ${ }^{2}$ Therefore, the role of conformational changes on proteinprotein binding is a topic of great interest, from both the computational and the experimental points of view. ${ }^{6-9} 14-3-3$ adapter proteins are an especially interesting case for the regulation of IDPs. $90 \%$ of their more than 250 partner proteins contain disordered regions with phosphorylationdependent 14-3-3 recognition motifs. ${ }^{10}$ Examples of such partner proteins are the tumor suppressor $\mathrm{p} 53,{ }^{11,12}$ the Alzheimer's and Parkinson's disease-related proteins Tau, ${ }^{13,14}$ and $\alpha$-Synuclein, ${ }^{15}$ the Cystic Fibrosis Transmembrane Conductance Regulator (CFTR), ${ }^{16-18}$ and the cell-cycle interconverting conformations. ${ }^{4,5}$ One functional advantage of

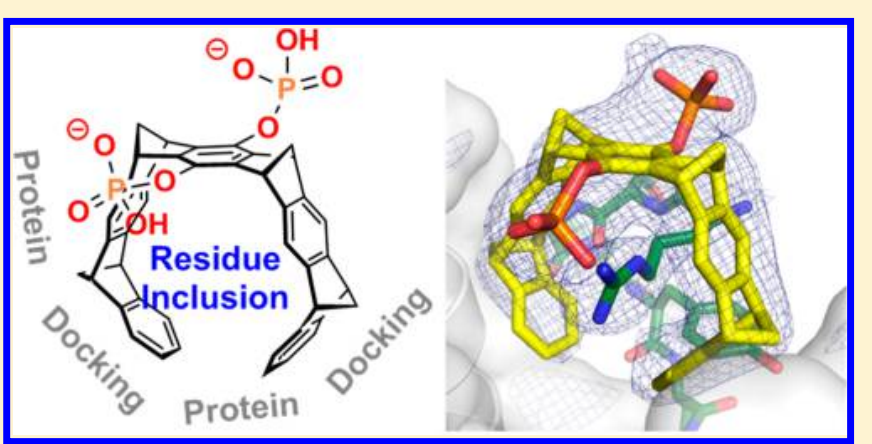

phosphatase cell-division-cycle $25 \mathrm{C}$ (Cdc25C). ${ }^{19,20}$ Upon binding to the rigid 14-3-3 protein, the recognition motifs undergo a disorder-to-order transition which might impose a specific functional fold of the IDP. ${ }^{21,22}$

Modulation of PPIs with small-molecules is one of the most promising new approaches in chemical biology and drug discovery. It provides unprecedented opportunities for the analysis of biological pathways and phenomena as well as novel approaches for therapeutic intervention in nearly every human disease. Whereas inhibition of PPIs is meanwhile a wellaccepted strategy with a number of convincing success stories, ${ }^{23}$ the opposite approach to stabilize regulatory protein complexes is considerably less explored. However, some natural products and a growing number of synthetic molecules mediate their physiological activity by stabilizing PPIs. ${ }^{24-28}$ Recently we demonstrated how the molecular tweezer CLR01 ${ }^{29,30}$ can inhibit 14-3-3 protein-protein interactions with partner proteins like C-Raf and Exoenzyme $S^{31}$ CLR01 is a supramolecular ligand tailored for lysine and arginine complex-

Received: August 8, 2017

Published: October 17, 2017 
ation $^{32}$ through a unique binding mode: the basic amino acid threads its side chain through the tweezer cavity and locks its side chain cation into a salt bridge with the tweezer hydrogen phosphate anion. ${ }^{33}$ No other amino acid residue is accommodated nor any cofactor is bound inside the well-defined cavity. In unfolded peptides, each lysine and arginine residue appears to be accessible, whereas on protein surfaces only the best accessible $\mathrm{R} / \mathrm{K}$ residues are reached. ${ }^{34-36}$ In the crystal structure an exposed lysine at position 214 on the upper rim of the 14-3-3 binding channel was thus complexed by a tweezer molecule, which prevented access of the partner protein. ${ }^{31}$

In principle, the convex aromatic sidewalls of the lysine binder represent another recognition face, suitable to target nonpolar flanks or grooves within the protein. Thus, nonpolar residues in the immediate vicinity of the complexed Lys 214 could be engaged in hydrophobic contacts with these external tweezer sidewalls and most likely further stabilize the complex. Conceptually this is called a "Janus" molecule, a term introduced in supramolecular chemistry by Lehn et al, originally describing a Roman god with two faces (Figure 1). ${ }^{37,38}$ If the

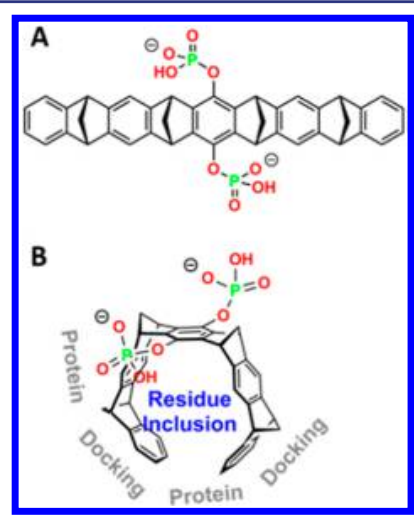

Figure 1. (A) Structure of the molecular tweezer CLR01. (B) The Janus-type molecular tweezer CLR01 offers two separate recognition sites for proteins: the aromatic cavity with flanking hydrogen phosphate anions accommodates a lysine or argine chain, whereas the convex exterior presents an apolar aromatic surface able to dock onto hydrophobic clefts on the target protein.

tweezers would, however, form a complex with a basic residue of the partner protein and simultaneously dock onto a nonpolar patch of the central 14-3-3 binding channel, the Janus binding mode would strengthen the protein-protein interaction. To analyze a potential PPI stabilizing activity we tested our molecular tweezers CLR01 in a biochemical assay in which a synthetic, labeled partner protein peptide binds to 14-3-3 leading to an increase in fluorescence polarization. Binding of peptides derived for example from C-Raf and Exoenzyme $S$ was inhibited in the presence of CLR01. ${ }^{31}$ In contrast to this, we found a stabilizing effect toward the binding of the recognition motif from Cdc25CpS216 to 14-3-3. Cdc25C is a dual specificity phosphatase which in the nucleus dephosphorylates and thereby activates cyclin-dependent kinases (Cdks), thus promoting cell cycle progression and proliferation. ${ }^{39}$ 14-3-3 proteins have been reported to bind to a recognition motif surrounding the phosphorylated serine 216 in $\mathrm{Cdc} 25 \mathrm{C}$ (Cdc25CpS216) and act as negative regulators of $\mathrm{Cdc} 25 \mathrm{C}$ nuclear import. ${ }^{20}$ 14-3-3 proteins are therefore functional inhibitors of this protein phosphatase which has been found to be overly active in many cancers. ${ }^{40}$ In the aforementioned biochemical assay, addition of CLR01 increased the apparent affinity of the Cdc25CpS216 peptide toward $14-3-3 \zeta$ around 20 -fold. The crystal structure of the ternary complex between 14-3-3 $\zeta$, the Cdc25CpS216 peptide and CLR01 revealed that the molecular tweezer is bound to R208 of the Cdc25C peptide

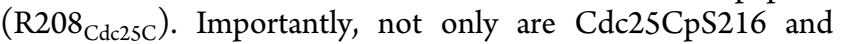
CLR01 simultaneously bound to the amphipathic groove of 143-3 , but also the number of residues of the peptide that can be identified in the electron density is more than doubled in the presence of CLR01. Finally, in silico studies allowed ration-

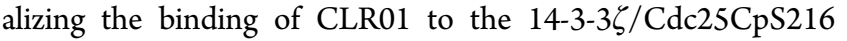
complex in solution with general implications for the targeted stabilization of PPI interfaces.

\section{RESULTS AND DISCUSSION}

Stabilization of the 14-3-3 $\zeta / C d c 25 C p S 216$ ProteinProtein Interaction. There are some intriguing features of small-molecule PPI stabilizers that qualify them as valuable complements of today's focus on inhibitors of PPIs or enzyme active sites, for example their uncompetitive nature and potential for higher specificity. They are furthermore valuable tools for structural biology studies of their in many cases transient target protein complexes. Natural product PPI stabilizers enabled the first crystallographic structure determination of their target protein complexes, e.g., forskolin stabilizing an adenylyl cyclase complex, ${ }^{41}$ brefeldin arresting the interaction of the small $G$ protein Arf with its guanine exchange factor Arf-GEF ${ }^{42}$ or fusicoccin acting on a 14-3-3 complex with the regulatory domain of the proton pump PMA2. ${ }^{43,44}$ On the basis of our previous structural finding how CLR01 inhibits the interaction of C-Raf and ExoS to 14-3-3, ${ }^{31}$ we aimed at elucidating if CLR01 could also show a stabilizing activity toward a 14-3-3 PPI. Hence we tested the effect of CLR01 in a biochemical assay toward a number of 14-3-3 $\zeta$ PPIs, for example, with p53, ${ }^{12} \mathrm{Cdc} 25 \mathrm{C},{ }^{19,20} \mathrm{Mlf1},{ }^{45} \mathrm{PAD}^{46},{ }^{46}$ TASK $3,{ }^{47}$ and Tau. ${ }^{14}$ Binding of fluorescein-labeled peptides derived from the aforementioned recognition motifs was tested in a fluorescence polarization (FP) assay to $14-3-3 \zeta$ in the absence and presence of CLR01. Only in the case of the Cdc25C peptide (Cdc25CpS216) an increase in apparent affinity was detected, from an initial $K_{\mathrm{d}}$ of $30.4 \mu \mathrm{M}$ in the DMSO control to $1.4 \mu \mathrm{M}$ in the presence of $250 \mu \mathrm{M}$ CLR01 (Figure 2). Thus, CLR01 was able to stabilize the peptideprotein complex by a factor of around 20. As a control, NMR and ITC titrations showed no direct interaction between CLR01 and the fluorescein label (see SI, Figure S5).

Biological precedence demonstrates that a 20 -fold affinity increase can be highly relevant: e.g., brefeldin A stabilizes its

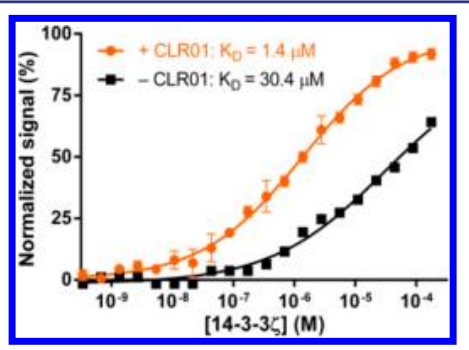

Figure 2. Binding of FAM-labeled Cdc25CpS216 to 14-3-3 $\zeta$. Titration of 14-3-3 $\zeta$ to FAM-Cdc25CpS216 resulted in the determination of a $K_{\mathrm{d}}$ of $30.4 \mu \mathrm{M}$, the same measurement in the presence of $250 \mu \mathrm{M}$ CLR01 produced an approximately 20 -fold lower apparent $K_{\mathrm{d}}$ of 1.4 $\mu \mathrm{M}$. 
target protein complex Arf1/Arf1GAP by a factor of 10 which is strong enough to entirely shut-down golgi-dependent transport in cells. ${ }^{48,49}$ Likewise, forskolin A stabilizes the interaction of the subunits of adenylyl cyclase with an $\mathrm{EC}_{50}$ of $10 \mu \mathrm{M}$. This is sufficient to significantly stimulate AC activity which leads to a strong increase in the concentration of cyclic AMP (cAMP). ${ }^{50,51}$ We have shown previously that the semisynthetic diterpene glycoside FC-THF stabilizes the interaction of 14-3-3 proteins with the potassium channel TASK3 around 19-fold which results in a $50 \%$ increase in plasma membrane localization. ${ }^{47}$ Similarly, the related natural products cotylenin $A$ and fusicoccin stabilize 14-3-3 binding with the protein kinase C-Raf ${ }^{48}$ and the chloride channel CFTR ${ }^{18}$ by factors of 17 and 9, respectively.

To further analyze this PPI stabilizing effect with another biophysical method we employed isothermal titration calorimetry (ITC) and titrated the nonlabeled Cdc25CpS216 peptide into $14-3-3 \zeta$ in the presence and absence of CLR01 (Figure 3).

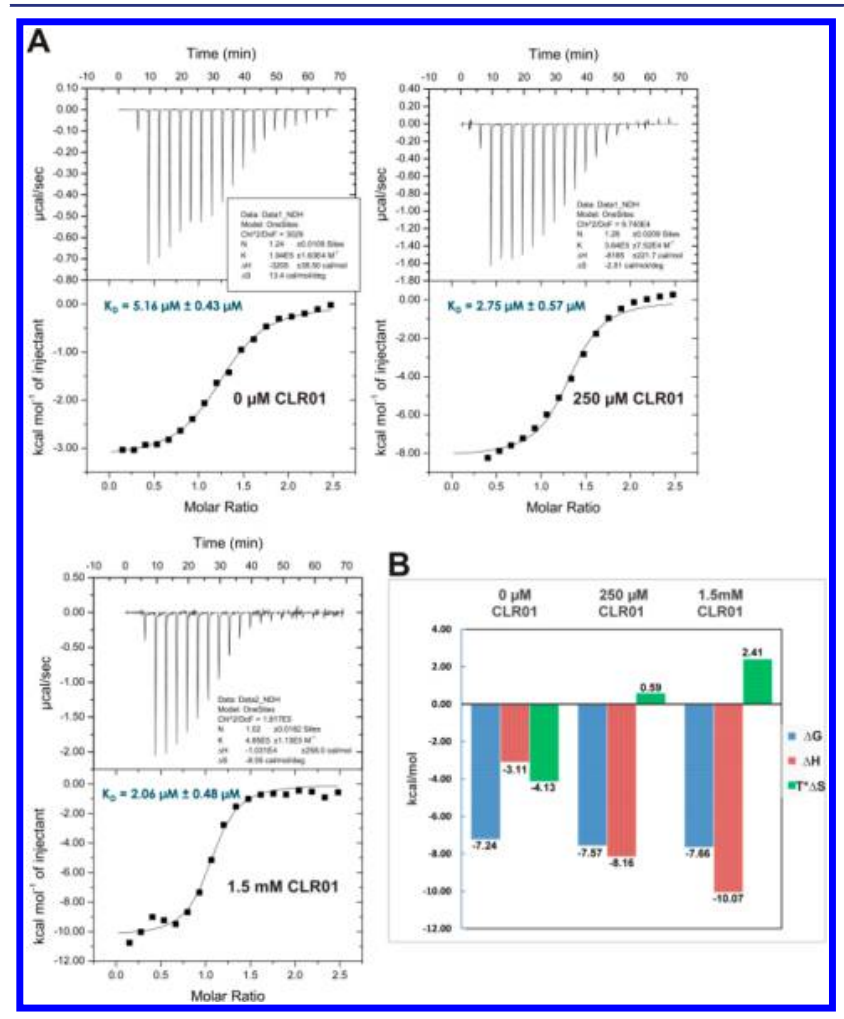

Figure 3. Binding of the $\mathrm{Cdc} 25 \mathrm{CpS} 216$ peptide to $14-3-3 \zeta$ measured by ITC. (A) Binding isotherms of titrating Cdc25CpS216 into 14-3-3 in the absence and presence of CLR01. (B) Thermodynamic parameters of the ITC titrations shown in A.

In the absence of CLR01 the resulting binding isotherm could be fitted in a 1:1 model and furnished an apparent $K_{\mathrm{d}}$ of 5.16 $\mu \mathrm{M}$ (Figure 3A). This value dropped to $2.75 \mu \mathrm{M}$ and finally to $2.06 \mu \mathrm{M}$ in the presence of $250 \mu \mathrm{M}$ or $1.5 \mathrm{mM}$ CLR01, testifying a moderate 2-3-fold reinforcement of the 14-3-3 $\zeta$ / Cdc25CpS216 interaction. However, the detailed thermodynamic analysis of this interaction reveals a highly interesting trend: increasing amounts of the molecular tweezer render the recognition event more enthalpically favorable and entropycostly, indicating a specific interaction and formation of a wellordered state (Figure 3B). As presented in the next section, this results agrees well with our protein crystallography data.
Crystal Structure of the $14-3-3 / C d c 25 C p S 216 / C L R 01$ Complex. We have previously shown how CLR01 inhibited the interaction of 14-3-3 with the partner proteins C-Raf and Exoenzyme $S$ by introducing a steric conflict in the proteinprotein interface. ${ }^{31}$ The resulting crystal structure showed the complex of 14-3-3 with CLR01 but without a partner protein peptide. To elucidate the structural basis of the stabilizing activity toward the $14-3-3 / \mathrm{Cdc} 25 \mathrm{CpS} 216$ interaction we performed soaking experiments with crystals of the binary complex. Thus, we first solved the crystal structure of 14-3-3 $\zeta$ in complex with Cdc25CpS216, a synthetic phosphopeptide derived from the 14-3-3 recognition motif surrounding Ser216 of Cdc25C. This phosphopeptide was also used for the aforementioned FP assays. The crystals of this complex displayed a 14-3-3 $\zeta$ dimer in the asymmetric unit and the electron density allowed building of seven residues of the Cdc25CpS216 peptide ( $2213_{\mathrm{Cdc} 25 \mathrm{C}}$ to $\left.\mathrm{E} 219_{\mathrm{Cdc25C}}\right)$. When these crystals were soaked with CLR01, additional electron density unambiguously accountable for CLR01 was found. Surprisingly, this density was not localized near or at a surface-exposed lysine of 14-3-3 $\zeta$ but directly in the amphipathic binding channel of 14-3-3 5 . This binding site is about $15 \AA$ away from $\mathrm{pS} 216_{\mathrm{Cdc} 25 \mathrm{C}}$. CLR01's electron density covered the entire molecule and allowed determining the position of both phosphate groups. More interestingly, we detected a significant amount of additional electron density that could easily be assigned to residues of the $\mathrm{Cdc} 25 \mathrm{CpS} 216$ peptide that are not seen in the density of the binary complex (Figure 4A,B). In total, 11

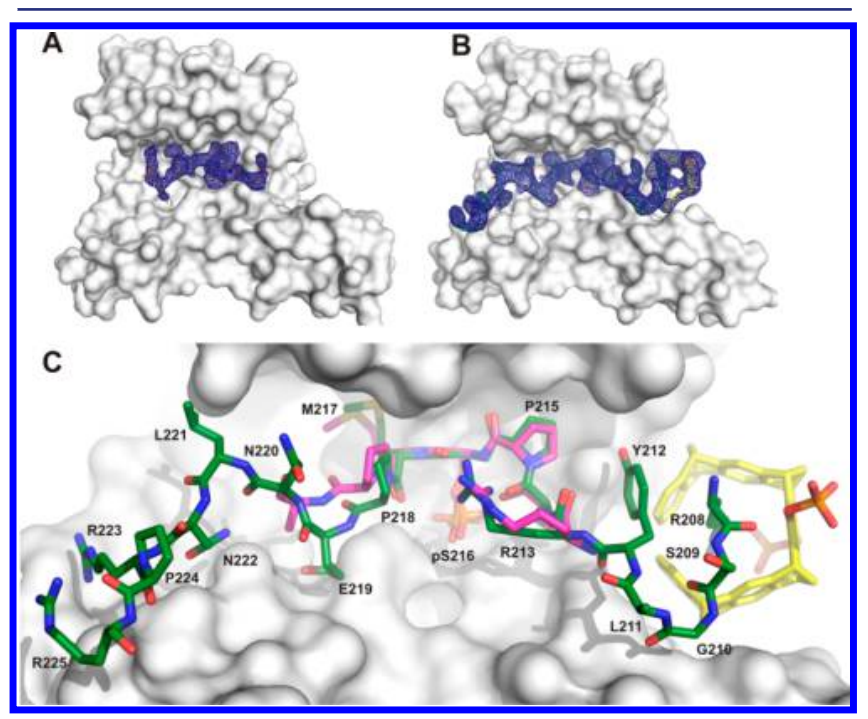

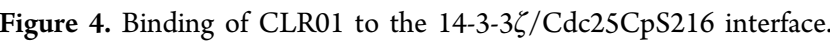
(A) Electron density $\left(2 F_{o}-F_{\mathcal{o}}\right.$ blue mesh) of the Cdc25CpS216 peptide bound to $14-3-3 \zeta$ (white solid surface). (B) Electron density as in (A) after soaking the crystals with CLR01. (C) Overlay of the Cdc25CpS216 peptide bound to $14-3-3 \zeta$ (white surface) in the absence (magenta sticks) and the presence (green sticks) of CLR01 (yellow sticks).

additional residues of the $\mathrm{Cdc} 25 \mathrm{CpS} 216$ peptide could be newly built (Figure 4C,A) thereby more than doubling the number of structurally defined residues of the interaction motif.

In fact, the Cdc25CpS216 peptide could be tracked $\mathrm{N}$ terminally to $\mathrm{R} 208_{\mathrm{Cdc} 25 \mathrm{C}}$ and C-terminally to $\mathrm{R} 225_{\mathrm{Cdc} 25 \mathrm{C}}$. At the $\mathrm{N}$-terminus, CLR01 establishes a direct contact with $\mathrm{Cdc} 25 \mathrm{CpS} 216$. The guanidinium cation of $\mathrm{R} 208_{\mathrm{Cdc} 25 \mathrm{C}}$ forms a salt bridge with the tweezer hydrogen phosphate group. It is 
simultaneously hidden inside the electron-rich cavity so that it undergoes extensive $\pi$-cation interactions with all five arenes (Figure 4B,C). This is also the first crystal structure of a complexed arginine side chain inside CLR01 confirming earlier computational predictions. ${ }^{35}$ In addition, the main chain of S209 ${ }_{\mathrm{Cdc25C}}$ and the side chain of $\mathrm{Y}_{212_{\mathrm{Cdc25C}}}$ are contributing a hydrophobic contact surface toward the apolar norbornadiene and benzene ring scaffold of the tweezer molecule. Simultaneously, CLR01 binds via its outer aromatic surface to a semicircular, mostly hydrophobic part of the $14-3-3 \zeta$ binding canyon contributed by $\mathrm{S} 63_{14-3-3 \zeta}$ and $\mathrm{S} 64_{14-3-3 \zeta}$, the side-chains of W59 $14-3-3 \zeta$ and $\mathrm{Y} 179_{14-3-3 \zeta}$ as well as the hydrocarbon parts of the side-chains of $\mathrm{R} 60_{14-3-3 \zeta}$ and $\mathrm{E} 180_{14-3-3 \zeta}$ (Figure 5C). While

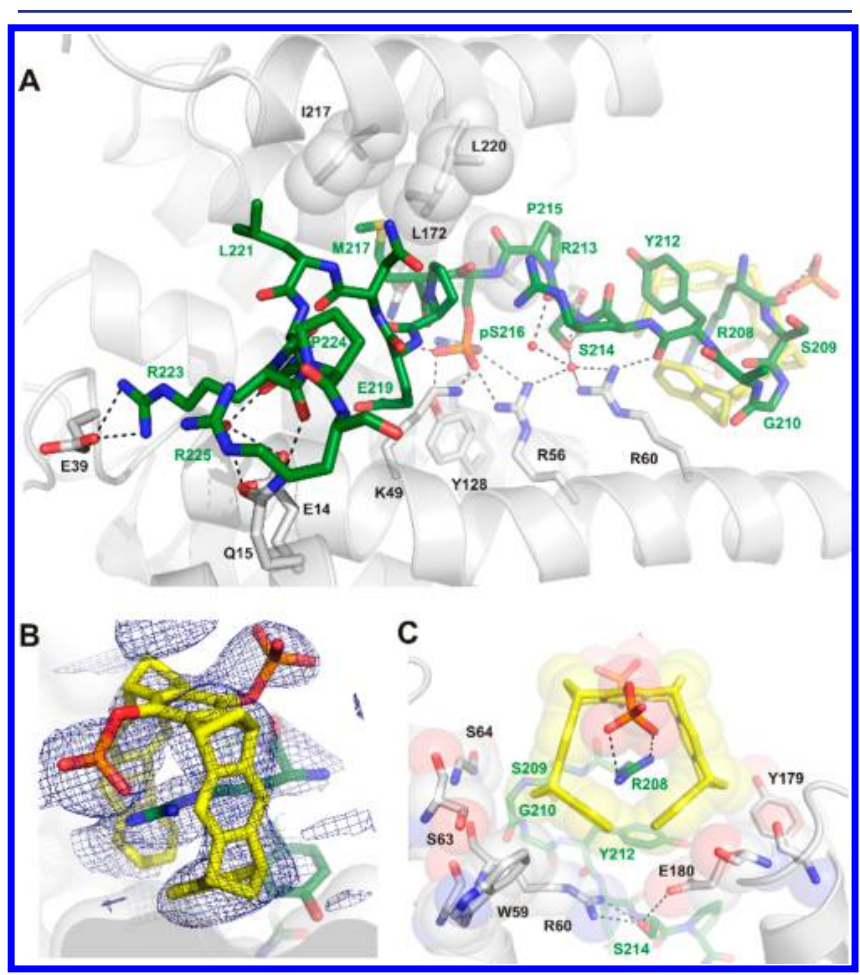

Figure 5. Crystal structure of $\mathrm{Cdc} 25 \mathrm{CpS} 216$ bound to $14-3-3 \zeta$ and CLR01. (A) Details of the interface of Cdc25CpS216 (green sticks) and $14-3-3 \zeta$ (white cartoon), stabilized by CLR01 (yellow sticks). Residues of $14-3-3 \zeta$ that are important for direct contacts with the Cdc25CpS216 peptide are shown as sticks, residues that contribute van-der-Waals contacts are shown as transparent spheres. Dotted lines indicate hydrogen bonds, water molecules are shown as red spheres. (B) Composite omit map (contoured at $1 \sigma$ ) electron density of CLR01 (yellow sticks) bound to 14-3-3 $\zeta$ (white cartoon and semitransparent surface) and Cdc25CpS216 (green sticks). (C) Details of the binding surface of the molecular tweezer accommodated in the amphipathic groove of 14-3-3 $\zeta$.

it is plausible that binding of CLR01 to the N-terminus of the peptide would stabilize this region and allow identification of the positions of additional residues, it was quite surprising that six more amino acids at the C-terminus of the Cdc25CpS216 peptide also became visible in the electron density. This can be rationalized by the number of additional contacts that are established between 14-3-3 $\zeta$ and $\mathrm{Cdc} 25 \mathrm{CpS} 216$ in the presence of CLR01 (Figure 4C, 5A). This way, CLR01 is able to stabilize the binding of the entire recognition motif and not just the region that is directly part of the tweezer's binding site. To provide structural corroboration of this observation and to analyze a possible influence of the peptide length, we synthesized a truncated version of the $\mathrm{Cdc} 25 \mathrm{C}$ peptide, which contained only the fragment $\mathrm{R} 207-\mathrm{R} 226_{\mathrm{Cdc} 25 \mathrm{C}}$ that underwent the disorder-to-order transition on $14-3-3 \zeta$ in the presence of CLR01.

We obtained crystals of the binary 14-3-3 $/$ Cdc25CpS216 (20mer) in the same space group and the same crystallographic cell as with the $14-3-3 \zeta / \mathrm{Cdc} 25 \mathrm{CpS} 216$ (38mer). After soaking these crystals for $24 \mathrm{~h}$ in a solution containing CLR01 and collecting a data set, the structure was solved to a resolution of $2.35 \AA$ A. The electron density allowed building of 17 residues (R208-P224) of the Cdc25CpS216 peptide with R208 being accommodated by CLR01 in the same way as in the structure with the 38mer peptide of Cdc25C (Figure S1). However, when comparing the binding of Cdc25CpS216 of the 20mer with the 38 mer peptide, some differences were observed in the C-terminal part (Figure S2): P224 is occupying another position and R225 is not visible in the electron density of the 20mer structure. Nevertheless, the two structures are comparable with a rmsd (root-mean-square deviation) of $1.828 \AA$ (all atoms included). The distribution of the temperature factors (B factors) also illustrates the similarities between the 20- and the 38 mer of Cdc25CpS216 (Figure S3). Moreover, we were able to identify an additional CLR01 molecule binding to $\mathrm{K} 74$ of $14-3-3 \zeta$ in the 20 mer crystal structure (Figure 6). This lysine is located at the beginning of

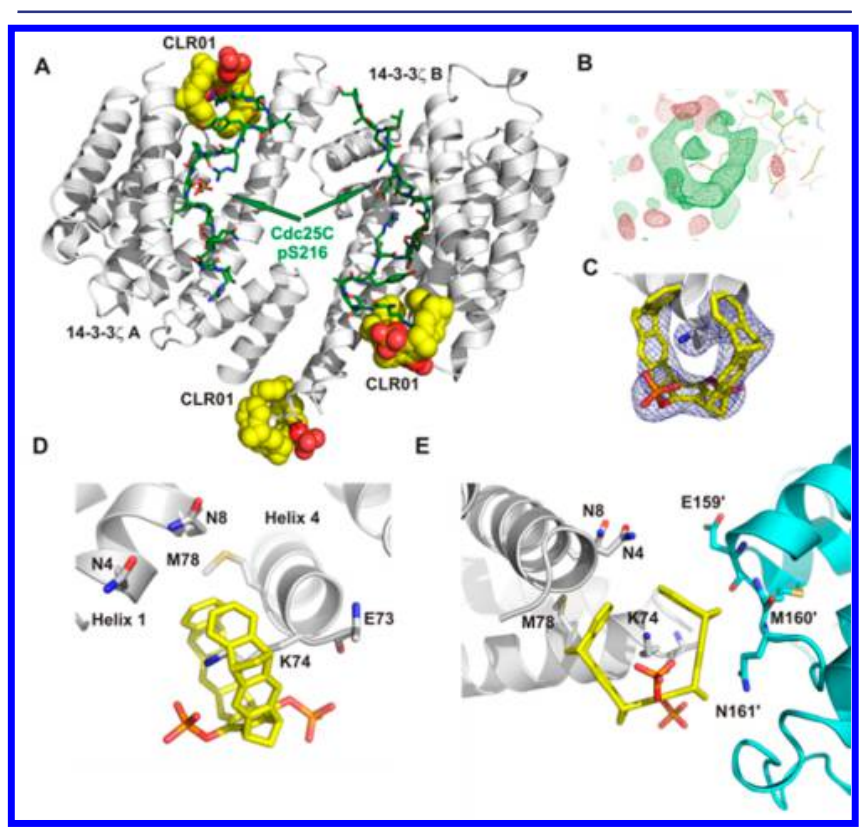

Figure 6. Binding of CLR01 to K74 of 14-3-3. (A) 14-3-3 $\zeta$ dimer (white cartoon) in complex with Cdc25CpS216 (20mer, green sticks) and three molecules of CLR01 (yellow spheres). (B) Unbiased $F_{\mathrm{o}}-F_{\mathrm{c}}$ electron density map (green mesh, contoured at $2.5 \sigma$ ) previous to include CLR01 in the model. (C) Final $2 F_{o}-F_{c}$ electron density map (blue mesh, contoured at $1 \sigma$ ). (D) Environment of CLR01 binding to K74 of one monomer of the 14-3-3 $\zeta$ dimer. (E) Stabilization of CLR01 binding to K74 by contacts established with a symmetryrelated 14-3-3 molecule (cyan cartoon and sticks).

helix 4 in one 14-3-3 monomer of the 14-3-3 $\zeta$ dimer and conveys a contact to helix 1 of the second 14-3-3 monomer (Figure 6A,D). Stabilization of CLR01 binding to K74 by a symmetry-related 14-3-3 unit (Figure 6E) is probably the reason why $\mathrm{CLRO1}_{\mathrm{K} 74}$ is visible in the electron density. 
In Silico Studies of Potential Binding Sites for CLR01. As shown by X-ray crystallography and FP measurements, in the context of the $14-3-3 \zeta / \mathrm{Cdc} 25 \mathrm{CpS} 216$ complex, CLR01 preferentially binds to $\mathrm{R} 208_{\mathrm{Cdc25C}}$ and furthermore stabilizes the protein-peptide complex by simultaneously establishing contacts to $14-3-3 \zeta$. To further investigate these processes in solution, we studied the preferred binding sites of CLR01 among all lysine and arginine residues found in both, Cdc25CpS216 and the $14-3-3 \zeta$ protein. Subsequently, we calculated the effect of CLR01 binding on the overall stability of the protein-peptide complex. To this end, molecular dynamics simulations (MD) in explicit solvent, quantum mechanics/ molecular mechanics ( $\mathrm{QM} / \mathrm{MM})$ and free energy calculations (free energy perturbation and umbrella sampling) were performed.

MDs of the ternary complexes (1:1:1 ratio) were carried out with CLR01 on each lysine or arginine residue, resulting in 35 simulation setups. QM/MM optimizations in explicit solvent were performed for snapshots from those MD trajectories with conserved inclusion complexes (Figures S8 and S9 and Supporting Information for additional details). These calculations indeed indicated $\mathrm{R} 208_{\mathrm{Cdc} 25 \mathrm{C}}$ as the most favorable arginine binding site for CLR01 in the peptide and confirmed the dual "Janus effect" of the tweezer in solution (Figure 7A,

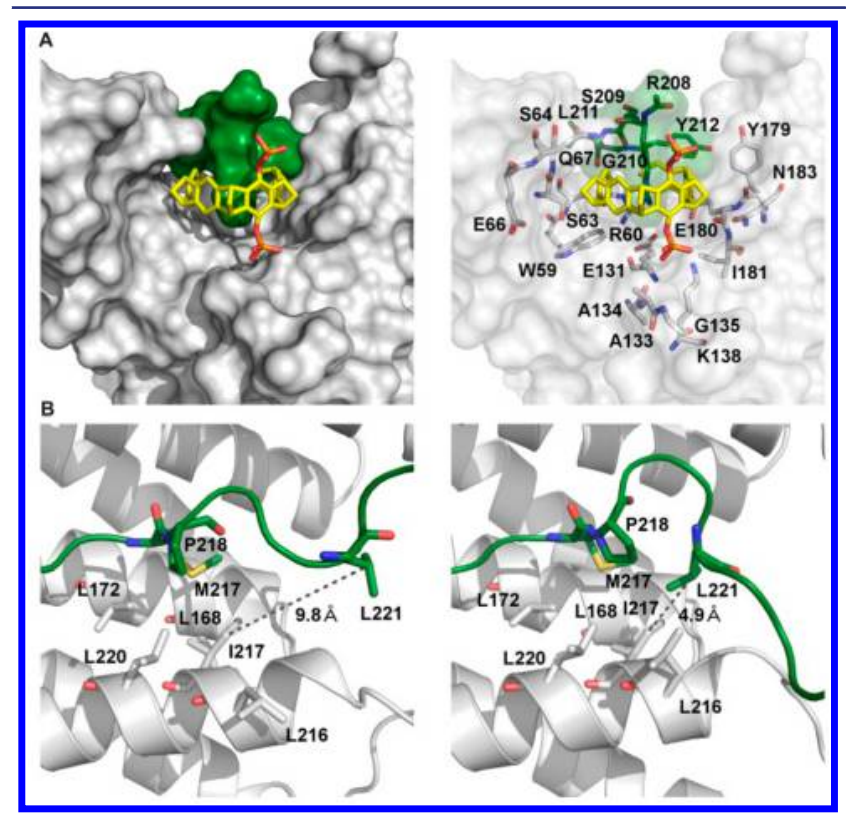

Figure 7. Binding of CLR01 to $\mathrm{R} 208_{\mathrm{Cdc} 25 \mathrm{C}}$ in solution. (A) CLR01

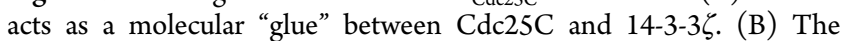
addition of CLR01 results in additional hydrophobic contacts between Cdc25C and 14-3-3 $\zeta$ (left, beginning of the simulation; right, hydrophobic collapse as the simulation progresses).

Table 1), in agreement with the crystal structure reported here. We also calculated free energy changes to compare the

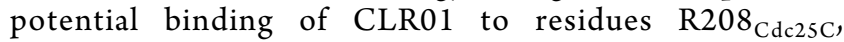

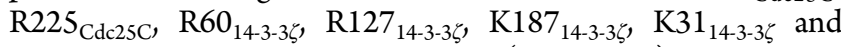
$\mathrm{K}_{44} 4_{14-36}$. The resulting values (Table S2) show that $\mathrm{R} 208_{\mathrm{Cdc25C}}$ is indeed superior to the best lysines regarding CLR01 binding. R208 combines guanidinium inclusion and an extra $\mathrm{C}=\mathrm{O} \cdots \mathrm{HO}-\mathrm{P}$ hydrogen bond with the additional stabilization inside the 14-3-3 channel. Such hydrogen bond stabilization is possible, because the second $\mathrm{p} K_{\mathrm{a}}$ value of the hydrogen phosphate group lies at 7.4 as determined potentio-
Table 1. Relative QM Energies for the Snapshots Optimized at the QM/MM Level of Theory (QM(B3LYP- D3/def2SVP)/CHARMM) ${ }^{a}$

\begin{tabular}{lcc}
\multicolumn{1}{c}{ residue } & relative QM energy $(\mathrm{kcal} / \mathrm{mol})$ & $\Delta G_{\text {bind }}(\mathrm{kcal} / \mathrm{mol})$ \\
$\mathrm{K} 187_{14-3-3 \zeta}$ & $0 \pm 3.6$ & $1.3 \pm 0.6$ \\
$\mathrm{~K} 3_{14-3-3 \zeta}$ & $0.9 \pm 8.7$ & $-3.0 \pm 0.4$ \\
$\mathrm{~K} 74_{14-3-3 \zeta}$ & $11.9 \pm 3.2$ & $-3.9 \pm 0.5$ \\
$\mathrm{R} 208_{\mathrm{Cdc25C}}$ & $0 \pm 2.1$ & $-4.5 \pm 0.3$ \\
$\mathrm{R} 0_{14-3-3 \zeta}$ & $8.8 \pm 10.9$ & $-4.8 \pm 0.4$ \\
$\mathrm{R} 127_{14-3-3 \zeta}$ & $21.0 \pm 1.0$ & $-2.8 \pm 0.6$ \\
$\mathrm{R} 225_{\mathrm{Cdc25C}}$ & $26.2 \pm 9.6$ & $-1.7 \pm 0.4$
\end{tabular}

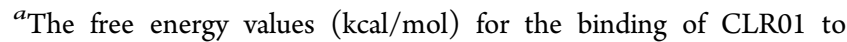
selected lysine and arginine residues in the $14-3-3 \zeta$ protein and Cdc25CpS216 were determined via alchemical transformation pathway. All calculations were performed on the ternary complex.

metrically (see Figure S6). At physiological pH, the tweezer phosphates thus carry one $\mathrm{OH}$ group, most likely engaged in hydrogen bonds. NMR and fluorescence titrations between CLR01 and the C-terminal part of the $\mathrm{Cdc} 25$ peptide $\left(\mathrm{S} 207_{\mathrm{Cdc25C}}-\mathrm{Y} 212_{\mathrm{Cdc} 25 \mathrm{C}}\right)$ additionally confirmed that $\mathrm{R} 208_{\mathrm{Cdc25C}}$ accommodation inside the tweezer cavity is energetically favorable $\left(30 \mu \mathrm{M} K_{\mathrm{d}}\right)$ (Figure S4). The crystallization experiments with the $20 \mathrm{mer}$ version of $\mathrm{Cdc} 25 \mathrm{CpS} 216$ also supported our prediction of K74 as another favored binding site (see crystal structure section and Table S2).

To complement the experimental studies in solution, we investigated the stabilizing effect of CLR01 on the proteinpeptide complex (umbrella sampling calculations details in the SI). For this purpose, we probed two different stoichiometries: (a) the binding of CLR01 to $\mathrm{R} 208_{\mathrm{Cdc} 25 \mathrm{C}}$ at the $14-3-3 \zeta$ /Cdc25CpS216 interface as observed in the crystal structure (1:1:1 complex) and (b) the binding of CLR01 in molar excess (1:1:9 complex), a situation that is typical for a titration experiment studying protein-ligand interactions. The calculated free energy change for the binding of $\mathrm{Cdc} 25 \mathrm{CpS} 216$ to $14-3-3 \zeta$ without the tweezers ( $1: 1$ complex) is $20.2 \mathrm{kcal} / \mathrm{mol}$ (Figure S10). In the presence of one CLR01 molecule bound to $\mathrm{R} 208_{\mathrm{Cdc} 25 \mathrm{C}}$, the free energy remains virtually unchanged $(19.3 \mathrm{kcal} / \mathrm{mol})$, but is increased to $22.4 \mathrm{kcal} / \mathrm{mol}$ when CLR01 is in excess. Another factor worth mentioning is that, during the molecular dynamics simulations, a hydrophobic collapse is observed when CLR01 is bound to R208 ${ }_{\mathrm{Cdc25C}}$ (Figure $7 \mathrm{~B}$, Supplementary Figure S11 and Supporting Information for details). This effect involves neighboring hydrophobic residues

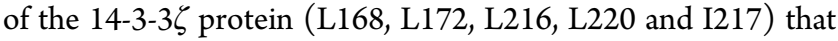
come in contact with the hydrophobic cluster formed by M217, P218, and L221 of Cdc25CpS216 (Figure 7B). The hydrophobic collapse-together with the increased number of interand intramolecular interactions - can contribute in solution to the disorder-to-order transition initiated by tweezer complexation on R208. It has to be taken into consideration that saturation of the critical R208 binding site of the tweezers competes with tweezer binding to a significant number of other lysines and arginines on the protein surface. Thus, the molecular glue effect cannot be expected to occur at a strict 1:1 ratio seen in the crystal structure, but requires a considerable tweezer excess. This is in line with the experimental results and our calculations.

Stabilization of Protein-Protein Interactions by Supramolecular Ligands. Here, we show for the first time 
how a supramolecular ligand can stabilize a 14-3-3 PPI. However, the underlying principles behind this stabilization have already been the subject of several previous studies. For example, systematic studies and databank mining by the Diederich group provided ample evidence for the extensive use of aromatic interactions to stabilize complexes between proteins and their binding partners. ${ }^{52,53}$ Quite often aromatic faces undergo dispersive and hydrophobic interactions with exposed residues on a protein surface.

One example is the complexation of thrombine's active site by a designed inhibitor, involved in numerous edge-to-face and $\mathrm{CH} / \pi$ contacts. $^{54,55}$ Another striking example is the 5-fold arene and arginine stacking exploited by the human growth hormone receptor. ${ }^{56}$ More recently, the structure of an aminothiazole inhibitor bound to $\beta$-ketoacyl-acyl carrier protein synthase (KAS) revealed parallel-displaced stacking interactions which rigidified the side chain of an involved phenylalanine. $^{57}$ Klebe et al. investigated aromatic aldol reductase inhibitors and discovered enthalpically favorable $\pi-\pi$ stacking interactions with a tryptophane. ${ }^{58}$ Our group very recently showed how the supramolecular ligand cucurbit [8] uril (Q8) can be used for a binary assembly platform using 14-3-3 and an ER $\alpha$-derived peptide $\mathrm{N}$-terminally labeled with a FGG motif. $^{59}$

In crystal structures between tetrasulfonatocalix[4]arene, a small supramolecular protein binder, and cytochrome c, Crowley et al. recently found a similar stabilization of hydrophobic (albeit rigid) protein-protein interfaces by docking of the nonpolar aromatic calixarene flanks. ${ }^{60}$ These "molecular glue" interactions were also discussed as facilitating protein crystallization. In our case, however, the PPI stabilization initiated by the tweezers, leads to remote conformational control inside the Cdc25CpS216 ligand across 18 residues, resulting in a "frozen" peptide geometry with numerous contacts toward the spacious rigid 14-3-3 channel. The RSMD values calculated for the peptide during the molecular dynamics simulations corroborate this assessment: Cdc25CpS216 is more rigid when CLR01 is interacting with R208 ${ }_{\mathrm{Cdc25C}}$ with respect to other simulations without tweezer or with one CLR01 molecule in another position (Figure S12). With its arginine side chain locked inside the tweezer's cavity, the entire peptide suffers from a substantial loss of degrees of freedom, which results in decreased on/off rates. Our computational studies probing CLR01's binding to all available lysines and arginines within the peptide/protein complex also suggest that $\mathrm{R} 208_{\mathrm{Cdc} 25 \mathrm{C}}$ is an optimal coordination site: it offers full steric accessibility for efficient arginine inclusion combined with perfect shape complementarity between the concave hydrophobic protein surface and the tweezer's lower aromatic region. These findings together with multivalent binding may explain why only the $14-3-3 \zeta / \mathrm{Cdc} 25 \mathrm{CpS} 216$ pair is stabilized by CLR01. If and how this PPI stabilization can be translated into a biological effect modulating the subcellular localization and physiological activity of $\mathrm{Cdc} 25 \mathrm{C}$ is subject of future collaborative research. In any case, the PPI-stabilizing activity of CLR01 adds to the impressive repertoire of supramolecular chemistry acting on proteins in general ${ }^{61}$ and 14-3-3 proteins in particular. $^{62}$

\section{CONCLUSION}

This study reveals in structural detail how the molecular tweezer CLR01 acts on the 14-3-3/Cdc25CpS216 proteinprotein interaction. Our investigation has delivered two key findings: (i) a supramolecular "Janus" ligand can bind simultaneously to a flexible peptidic PPI recognition motif and a well-structured adapter protein, and (ii) this binding fills a gap in the protein-protein interface, "freezes" one of the conformational states of the intrinsically disordered protein partner and enhances the apparent affinity of the interaction. It should be emphasized that the conformational restraint exerted on the Cdc25CpS216 peptide by the tweezer complexation significantly lowers the overall complex entropy. This value will be subtracted from the final free energy gain inside the ternary complex, which suggests that the stabilizing molecular glue effect is much larger than the measured $\mathrm{kcal} / \mathrm{mol}$ from the FP experiment. This important consideration also explains why there is no calculated total free energy gain for a single complexed CLR01 molecule.

Since many regulatory PPIs are characterized by such "disorder-to-order" transitions, the implications of this work go beyond the important class of the hub protein 14-3-3. They rather exemplify how supramolecular chemistry can in principle be employed to modulate the interaction of disordered proteins or intrinsically disordered protein regions with their partner proteins. We will in the future use libraries of modified tweezers to identify further peptide/protein pairs for efficient PPI stabilization. ${ }^{34}$ Small peptidic or artificial recognition elements attached to one of the phosphate esters on CLR01 may further noncovalently link the partner proteins and strengthen selected protein-protein interactions.

\section{METHODS}

Crystallization. For complex formation, the $14-3-3 \zeta$ protein $(22$ $\mathrm{mg} / \mathrm{mL}$ ) was mixed with the Cdc25C peptide $\left({ }^{207}\right.$ SRSGLYRSP-pSMPENLNRPRLKQVEKFKDNTIPDKVKKK ${ }^{244}$ ) in a molar ratio of $1: 1.5$ and incubated overnight at $4{ }^{\circ} \mathrm{C}$. Initial screens were performed by using NeXtal Screens (Qiagen). Three dimensional rod-like crystals grew within 4 weeks in the following conditions: $0.17 \mathrm{M}$ Ammonium acetate; 0.085 M Sodium citrate $\mathrm{pH}$ 5.6; 25.5\% (w/v) PEG 4000; $15 \%$ (v/v) Glycerol. The crystals could directly be flash-cooled in liquid nitrogen. For soaking experiments, the Na-tweezer salt was added to crystal-containing drops and incubated for $24 \mathrm{~h}$.

Data Collection and Structure Determination. Data was collected at the Paul Scherrer Institut (PSI) in Villigen, Switzerland at the PXII-X10SA beamline for protein crystallography. Diffraction data was analyzed using the XDS14 software. ${ }^{63}$ Molecular replacement and refinement steps were carried out with the CCP4 package. Model building was performed with COOT. ${ }^{64}$ Crystal structures were deposited in the Protein Data Bank.

Fluorescence Polarization (FP) Measurements. Fluorescence anisotropy based affinity measurements were performed using a filterbased microplate reader (Tecan Infinite F500) with a fluorescein filter set $\left(\lambda \lambda_{\mathrm{ex}}: 485 \mathrm{~nm}(20 \mathrm{~nm}), \lambda_{\mathrm{em}}: 535 \mathrm{~nm}(25 \mathrm{~nm})\right.$ and an integration time of $50 \mu \mathrm{s}$ in black, nonbinding surface, round-bottom 384 microwell plates (Corning \#3676). All measurements were performed using $100 \mathrm{nM}$ fluorescein amidite-labeled Cdc25pS216 peptide SRSGLYRSP(pS)MPENLNRPRLKQVEKFKDNTIPDK-VKKK-FAM in a buffer containing $10 \mathrm{mM}$ HEPES, pH 7.4, $150 \mathrm{mM} \mathrm{NaCl}, 0.1 \%$ (v/v) Tween 20 , and $0.1 \%(\mathrm{w} / \mathrm{v})$ BSA. First, the peptides were titrated with His-14-3-3 $\zeta$ in order to determine the (control) $K_{\mathrm{d}}$-value and select an appropriate concentration for the subsequent modulation experiments. For the determination of apparent $K_{\mathrm{d}}$-values a solution comprising $100 \mathrm{nM}$ of the $\mathrm{Cdc} 25 \mathrm{CpS} 216$ and $250 \mu \mathrm{M}$ of the tweezer molecule was titrated with the $14-3-3 \zeta$ protein. The measured anisotropy values were normalized using the maximum of the signal as $100 \%$ and plotted against the logarithmic protein concentration. To obtain $K_{\mathrm{d}}$-values the resulting curve was fitted to the "One-Site total binding" model using GraphPad Prism 5.03 for Windows (GraphPad Software Inc., CA, USA). 
Isothermal Titration Calorimetry (ITC). The ITC measurements were performed on a Malvern MicroCal iTC200. The cell contained $0.1 \mathrm{mM} 14-3-3 \zeta$ and the syringe contained a peptide solution at 1.26 $\mathrm{mM}$, both in ITC buffer [25 mM Hepes, $\mathrm{pH} 7.4,100 \mathrm{mM} \mathrm{NaCl}, 10$ $\mathrm{mM} \mathrm{MgCl}, 0.5 \mathrm{mM}$ Tris(2-carboxyethyl)-phosphin]. For the PPI stabilization experiments, the cell contained $0.1 \mathrm{mM} 14-3-3 \zeta$ and either $0.25 \mathrm{mM}$ or $1.50 \mathrm{mM}$ CLR01. The experiments were performed in triplicates and consisted of a series of $182-\mu \mathrm{L}$ titrations performed at $25^{\circ} \mathrm{C}$ (reference power, $5 \mu \mathrm{Cal} / \mathrm{s}$; initial delay, $180 \mathrm{~s}$; stirring speed, $750 \mathrm{rpm}$; spacing, $200 \mathrm{~s}$ ).

Computational Details. The initial coordinates used for the computational studies ( $\mathrm{MD}$ simulations, $\mathrm{QM} / \mathrm{MM}$ calculations, free energy calculations) were taken from the crystal structure reported here of the complex formed by 14-3-3 All MD simulations were performed using the NAMD 2.9 program. $^{65}$ The CHARMM27 force field with CMAP corrections was used for all atoms. $^{6,67}$ The parameters of CLR01 were obtained with the SwissParam server ${ }^{68}$ and previously tested by us. ${ }^{31,35,69}$ For the $\mathrm{QM} / \mathrm{MM}$ optimizations, Turbomole $6.4^{70}$ was used for the QM region and DL_POLY ${ }^{71}$ as driver of the CHARMM27 force field (MM region). The geometry optimizations were performed at the B3LYPD3/def2-SVP//CHARMM27 level of theory using the hybrid delocalized internal coordinates (HDLC) optimizer as implemented in ChemShell v3.5. ${ }^{72}$ The entire tweezer molecule and part of the lysine or arginine side chain were chosen as the QM region. The free energies of binding of CLR01 to the most favored lysine and arginine residues (as determined by QM/MM calculations) were computed via the alchemical transformation pathway. ${ }^{73}$ Additionally, umbrella sampling simulations ${ }^{74}$ were performed to investigate the effect of the tweezers on the binding of Cdc25CpS216 to 14-3-3 $\zeta$. The final PMF profiles were obtained using WHAM. $^{75}$ See Supporting Information for more details of the calculations.

\section{ASSOCIATED CONTENT}

\section{S Supporting Information}

The Supporting Information is available free of charge on the ACS Publications website at DOI: 10.1021/jacs.7b07939.

X-ray crystallography table, additional figures protein crystallography, investigation of model systems in aqueous buffer, extended computational details (PDF)

\section{AUTHOR INFORMATION}

\section{Corresponding Authors}

*elsa.sanchez-garcia@uni-due.de

*thomas.schrader@uni-due.de

*c.ottmann@tue.nl, christian.ottmann@uni-due.de

\section{ORCID $\odot$}

Luc Brunsveld: 0000-0001-5675-511X

Elsa Sanchez-Garcia: 0000-0002-9211-5803

Thomas Schrader: 0000-0002-7003-6362

Christian Ottmann: 0000-0001-7315-0315

\section{Author Contributions}

${ }^{\#}$ S.M., K.B.-R., and A.S. contributed equally.

\section{Notes}

The authors declare no competing financial interest.

\section{ACKNOWLEDGMENTS}

$\mathrm{X}$-ray data collection was performed at the Swiss Light Source, beamline X10SA, Paul Scherrer Institute, Villigen, Switzerland. We thank the beamline staff and Mathias Müller, Marina Keul, Emerich Mihai Gazdag and Puneet Juneja for help with the data collection. This work was supported by the Collaborative Research Centre 1093, funded by the Deutsche Forschungsgemeinschaft (DFG), and The Netherlands Organization for
Scientific Research via Gravity program 024.001.035 and VICI grant 016.150.366. E.S-G. acknowledges the support of the Boehringer Ingelheim Foundation (Plus-3 Program), of the Cluster of Excellence RESOLV (EXC 1069) funded by the DFG, and the computational time provided by the Computing and Data Facility of the Max Planck Society.

\section{REFERENCES}

(1) Dunker, A. K.; Uversky, V. N. Nat. Chem. Biol. 2008, 4, 229.

(2) Dyson, H. J.; Wright, P. E. Nat. Rev. Mol. Cell Biol. 2005, 6, 197.

(3) Ward, J. J.; Sodhi, J. S.; McGuffin, L. J.; Buxton, B. F.; Jones, D. T. L. Mol. Biol. 2004, 337, 635.

(4) Meszaros, B.; Simon, I.; Dosztanyi, Z. Phvs. Biol. 2011, 8, 035003.

(5) Forman-Kay, J. D.; Mittag, T. Structure 2013, 21, 1492.

(6) Janin, J. Protein Sci. 2014, 23, 1813.

(7) Chipot, C. WIREs Comput. Mol. Sci. 2014, 4, 71.

(8) Noskov, S. Y.; Lim, C. Biophvs. I. 2001, 81, 737.

(9) Ma, X. H.; Wang, C. X.; Li, C. H.; Chen, W. Z. Protein Eng. Des. Sel. 2002, 15, 677 .

(10) Bustos, D. M.; Iglesias, A. A. Proteins: Struct., Funct., Genet. 2006, 63, 35.

(11) Rajagopalan, S.; Jaulent, A. M.; Wells, M.; Veprintsev, D. B.; Fersht, A. R. Nucleic Acids Res. 2008, 36, 5983.

(12) Schumacher, B.; Mondry, J.; Thiel, P.; Weyand, M.; Ottmann, C. FEBS Lett. 2010, 584, 1443.

(13) Sadik, G.; Tanaka, T.; Kato, K.; Yamamori, H.; Nessa, B. N.; Morihara, T.; Takeda, M. I. Neurochem. 2009, 10, 33.

(14) Joo, Y.; Schumacher, B.; Landrieu, I.; Bartel, M.; Smet-Nocca, C.; Jang, A.; Choi, H. S.; Jeon, N. L.; Chang, K. A.; Kim, H. S.; Ottmann, C.; Suh, Y. H. FASEB I. 2015, 29, 4133.

(15) Plotegher, N.; Kumar, D.; Tessari, I.; Brucale, M.; Munari, F.; Tosatto, L.; Belluzzi, E.; Greggio, E.; Bisaglia, M.; Capaldi, S.; Aioanei, D.; Mammi, S.; Monaco, H. L.; Samo, B.; Bubacco, L. Hum. Mol. Genet. 2011, 23, 5615-29.

(16) Liang, X.; Da Paula, A. C.; Bozóky, Z.; Zhang, H.; Bertrand, C. A.; Peters, K. W.; Forman-Kay, J. D.; Frizzell, R. A. Mol. Biol. Cell 2011, 23, 996.

(17) Bozoky, Z.; Krzeminski, M.; Muhandiram, R.; Birtley, J. R.; AlZahrani, A.; Thomas, P. J.; Frizzell, R. A.; Ford, R. C.; Forman-Kay, J. D. Proc. Natl. Acad. Sci. U. S. A. 2013, 110, E4427.

(18) Stevers, L. M.; Lam, C. V.; Leysen, S. F. R.; Meijer, F. A.; van Scheppingen, D. S.; de Vries, R. M. J. M.; Carlile, G. W.; Milroy, L. G.; Thomas, D. Y.; Brunsveld, L.; Ottmann, C. Proc. Natl. Acad. Sci. U. S. A. 2016, 113, E1152-61.

(19) Peng, C. Y.; Graves, P. R.; Thoma, R. S.; Wu, Z.; Shaw, A. S.; Piwnica-Worms, H. Science 1997, 277, 1501.

(20) Lopez-Girona, A.; Furnari, B.; Mondesert, O.; Russell, P. Nature 1999, 397, 172.

(21) Wright, P. E.; Dyson, H. I. Curr. Opin. Struct. Biol. 2009, 19, 31.

(22) Uversky, V. N. Biochim. Biophys. Acta, Proteins Proteomics 2013, $1834,932$.

(23) Arkin, M. R.; Tang, Y.; Wells, J. A. Chem. Biol. 2014, 21, 1102.

(24) Thiel, P.; Kaiser, M.; Ottmann, C. Angew. Chem., Int. Ed. 2012, $51,2012$.

(25) Milroy, L. G.; Grossmann, T. N.; Hennig, S.; Brunsveld, L.; Ottmann, C. Chem. Rev. 2014, 114, 4695.

(26) Giordanetto, F.; Schäfer, A.; Ottmann, C. Drug Discovery Todav 2014, 19, 1812.

(27) Bier, D.; Thiel, P.; Briels, J.; Ottmann, C. Prog. Biophvs. Mol. Biol. 2014, 119, 10.

(28) Andrei, S. A.; Sijbesma, E.; Hann, M.; Davis, J.; O’Mahony, G.; Perry, M. W. D.; Karawajczyk, A.; Eickhoff, J.; Brunsveld, L.; Doveston, R. G.; Milroy, L. G.; Ottmann, C. Expert Opin. Drug Discovery 2017, 12, 925.

(29) Zimmerman, S. C. Top. Curr. Chem. 1993, 165, 71.

(30) Zimmerman, S. C. Beilstein J. Org. Chem. 2016, 12, 125. 
(31) Bier, D.; Rose, R.; Bravo-Rodriguez, K.; Bartel, M.; RamirezAnguita, J. M.; Dutt, S.; Wilch, C.; Klärner, F. G.; Sanchez-Garcia, E.; Schrader, T.; Ottmann, C. Nat. Chem. 2013, 5, 234.

(32) Fokkens, M.; Schrader, T.; Klärner, F. G. L. Am. Chem. Soc. 2005, 127, 14415

(33) Klärner, F. G.; Kahlert, B.; Nellesen, A.; Zienau, J.; Ochsenfeld, C.; Schrader, T. I. Am. Chem. Soc. 2006, 128, 4831.

(34) Klärner, F. G.; Schrader, T. Acc. Chem. Res. 2013, 46, 967.

(35) Dutt, S.; Wilch, C.; Gersthagen, T.; Talbiersky, P.; BravoRodriguez, K.; Hanni, M.; Sánchez-García, E.; Ochsenfeld, C.; Klärner, F. G.; Schrader, T. I. Org. Chem. 2013, 78, 6721.

(36) Molzan, M.; Kasper, S.; Röglin, L.; Skwarczynska, M.; Sassa, T.; Inoue, T.; Breitenbuecher, F.; Ohkanda, J.; Kato, N.; Schuler, M.; Ottmann, C. ACS Chem. Biol. 2013, 8, 1869.

(37) Marsh, A.; Nolen, E. G.; Gardinier, K. M.; Lehn, J. M. Tetrahedron Lett. 1998, 35, 97.

(38) Kimizuka, N.; Fujikawa, S.; Kuwahara, H.; Kunitake, T.; Marsh, A.; Lehn, J. M. I. Chem. Soc. Chem. Commun. 1995, 2103.

(39) Galaktionov, K.; Beach, D. Cell 1991, 67, 1181-1194.

(40) Kristjansdottir, K.; Rudolph, J. Chem. Biol. 2004, 11, 10431051 .

(41) Tesmer, J. J.; Sunahara, R. K.; Gilman, A. G.; Sprang, S. ․․ Science 1997, 278, 1907.

(42) Renault, L.; Guibert, B.; Cherfils, J. Nature 2003, 426, 525.

(43) Ottmann, C.; Marco, S.; Jaspert, N.; Marcon, C.; Schauer, N.; Weyand, M.; Vandermeeren, C.; Duby, G.; Boutry, M.; Wittinghofer, A.; Rigaud, J.-L.; Oecking, C. Mol. Cell 2007, 25, 427.

(44) Molzan, M.; Ottmann, C. I. Mol. Biol. 2012, 423, 486.

(45) Molzan, M.; Weyand, M.; Rose, R.; Ottmann, C. FEBS I. 2012, $97,563$.

(46) Rose, R.; Rose, M.; Ottmann, C. I. Struct. Biol. 2012, 180, 65.

(47) Anders, C.; Higuchi, Y.; Koschinsky, K.; Bartel, M.; Schumacher, B.; Thiel, P.; Nitta, H.; Preisig-Müller, R.; Schlichthörl, G.; Renigunta, V.; Ohkanda, J.; Daut, J.; Kato, N.; Ottmann, C. Chem. Biol. 2013, 20, 583.

(48) Peyroche, A.; Antonny, B.; Robineau, S.; Acker, J.; Cherfils, J.; Jackson, C. L. Mol. Cell 1999, 3, 275.

(49) Zeeh, J. C.; Zeghouf, M.; Grauffel, C.; Guibert, B.; Martin, E.; Dejaegere, A.; Cherfils, J. I. Biol. Chem. 2006, 281, 11805.

(50) Seamon, K. B.; Padgett, W.; Daly, J. W. Proc. Natl. Acad. Sci. U. S. A. 1981, 78, 3363 .

(51) Sunahara, R. K.; Dessauer, C. W.; Whisnant, R. E.; Kleuss, C.; Gilman, A. G. L. Biol. Chem. 1997, 272, 22265.

(52) Molzan, M.; Kasper, S.; Röglin, L.; Skwarczynska, M.; Sassa, T.; Inoue, T.; Breitenbuecher, F.; Ohkanda, J.; Kato, N.; Schuler, M.; Ottmann, C. ACS Chem. Biol. 2013, 8, 1869.

(53) Meyer, E. A.; Castellano, F. R.; Diederich, F. Angew. Chem., Int. Ed. 2003, 42, 2010.

(54) Salonen, L. M.; Ellermann, M.; Diederich, F. Angew. Chem., Int. Ed. 2011, 50, 4808.

(55) Obst, U.; Banner, D. W.; Weber, L.; Diederich, F. Chem. Biol. 1997, 4, 287-295.

(56) de Vos, A. M.; Ultsch, M.; Kossiakoff, A. A. Science 1992, 255, 306-312.

(57) Pappenberger, G.; Schulz-Gasch, T.; Kusznir, E.; Müller, F.; Hennig, M. Acta Crystallogr., Sect. D: Biol. Crustallogr. 2007, 63, 12081216.

(58) Steuber, H.; Heine, A.; Klebe, G. I. Mol. Biol. 2007, 368, 618638.

(59) de Vink, P. J.; Briels, J. M.; Schrader, T.; Milroy, L. G.; Brunsveld, L.; Ottmann, C. Angew. Chem. Int. Ed. 2017, 56, 8998.

(60) McGovern, R. E.; Fernandes, H.; Khan, A. R.; Powera, N. P.; Crowley, P. B. Nat. Chem. 2012, 4, 527.

(61) van Dun, S.; Ottmann, C.; Milroy, L. G.; Brunsveld, L. I. Am. Chem. Soc. 2017, 139, 13960.

(62) Stevers, L. M.; de Vries, R. M.; Doveston, R. G.; Milroy, L. G.; Brunsveld, L.; Ottmann, C. J. Med. Chem. 2017, DOI: 10.1021/ acs.jmedchem.7b00574.
(63) Kabsch, W. Acta Crystallogr., Sect. D: Biol. Crystallogr. 2010, D66, 125

(64) Emsley, P.; Cowtan, K. Acta Crystallogr., Sect. D: Biol. Crustallogr. 2004, 60, 2126.

(65) Phillips, J. C.; Braun, R.; Wang, W.; Gumbart, J.; Tajkhorshid, E.; Villa, E.; Chipot, C.; Skeel, R. D.; Kalé, L.; Schulten, K. I. Comput. Chem. 2005, 26, 1781.

(66) Mackerell, A. D.; Feig, M.; Brooks, C. L. I. Comput. Chem. 2004, 25, 1400.

(67) MacKerell, A. D.; Bashford, D.; Bellott, M.; Dunbrack, R. L.; Evanseck, J. D.; Field, M. J.; Fischer, S.; Gao, J.; Guo, H.; Ha, S.; Joseph-McCarthy, D.; Kuchnir; Kuczera, K.; Lau, F. T.; Mattos, C.; Michnick, S.; Ngo, T.; Nguyen, D. T.; Prodhom, B.; Reiher, W. E.; Roux, B.; Schlenkrich, M.; Smith, J. C.; Stote, R.; Straub, J.; Watanabe, M.; Wiórkiewicz-Kuczera, J.; Yin, D.; Karplus, M. I. Phvs. Chem. B 1998, 102 (18), 3586.

(68) Zoete, V.; Cuendet, M. A.; Grosdidier, A.; Michielin, O. I. Comput. Chem. 2011, 32 (11), 2359.

(69) Lopes, D. H.; Attar, A.; Nair, G.; Hayden, E. Y.; Du, Z.; McDaniel, K.; Dutt, S.; Bravo-Rodriguez, K.; Mittal, S.; Klärner, F. G.; Wang, C.; Sanchez-Garcia, E.; Schrader, T.; Bitan, G. ACS Chem. Biol. $2015,10,1555$.

(70) Ahlrichs, R.; Bär, M.; Häser, M.; Horn, H.; Kölmel, C. Chem. Phvs. Lett. 1989, 162, 165.

(71) Smith, W.; Forester, T. R. I. Mol. Graphics 1996, 14, 136.

(72) Sherwood, P.; de Vries, A. H.; Guest, M. F.; Schreckenbach, G.; Lennartz, C. I. Mol. Struct.: THEOCHEM 2003, 632, 1-28.

(73) Gumbart, J. C.; Roux, B.; Chipot, C. I. Chem. Theory Comput. 2013, 9 (1), 794-802.

(74) Torrie, G. M.; Valleau, J. P. I. Comput. Phys. 1977, 23, 187.

(75) Kumar, S.; Rosenberg, J. M.; Bouzida, D.; Swendsen, R. H.; Kollman, P. A. I. Comput. Chem. 1992, 13, 1011. 